\title{
Metastatic Kaposi's Sarcoma Causing Gastrointestinal Bleeding: Report of an Unusual Case
}

\begin{abstract}
Mao-Yu Huang ${ }^{1,2}$, Feng-Cheng Liu ${ }^{3}$, Hong-Wei $\mathrm{Gao}^{4}$ and Tien-Yu Huang ${ }^{2,5 *}$

${ }^{1}$ Division of Gastroenterology, Department of Internal Medicine, Taoyuan Armed Forces General Hospital, Taiwan

${ }^{2}$ Division of Gastroenterology, Department of Internal Medicine, Tri-Service General Hospital, Taiwan

${ }^{3}$ Division of Rheumatology/ Immunology and Allergy, TriService General Hospital, Taiwan

${ }^{4}$ Department of Pathology, Tri-Service General Hospital, Taiwan

${ }^{5}$ Taiwan Association for the Study of Small Intestinal Diseases, Taiwan

*Corresponding author: Tien-Yu Huang, Division of Gastroenterology, Department of Medicine, Tri-Service General Hospital, National Defense Medical Center, Taipei, No. 325, Section 2, Cheng-Kung Road, Neihu 114, Taipei, Taiwan
\end{abstract}

Received: December 01, 2016; Accepted: February 07, 2017; Published: February 09, 2017

\section{Introduction}

Kaposi sarcoma (KS) is a multifocal low-grade vascular malignancy that is associated with Human Herpesvirus-8 (HHV8) infection [1]. The first description of this tumor dates back to 1872 and was made by Dr. Moritz Kaposi, a Hungarian dermatologist who described 5 cases of "idiopathic multiple pigmented sarcomas of the skin". Four distinct forms have been characterized including classic KS, endemic or African KS, organ transplant-associated KS, and AIDS-related KS $[2,3] . \mathrm{KS}$ is the most common malignancy in patients with AIDS, especially among men who have sex with men (MSM) and low CD4 count of $<100$ cell/ $\mu \mathrm{L}$. It also develops in the setting of advanced immune suppression frequently $[3,4,5]$.

Skin lesions are the most common manifestation in patients with KS, typically the skin of the lower extremities, face, trunk and genitalia. Visceral involvement is also frequent, affecting most commonly the gastrointestinal tract (40\%) and lungs (30\%) [6,7]. Most patients with GI involvement are asymptomatic, and therefore, most visceral KS remain unidentified [4]. However, it may present with nonspecific symptoms such as weight loss, diarrhea, abdominal pain and Gastrointestinal bleeding [8]. Obstruction, perforation, intussusception, and protein-losing enteropathy are infrequent complications associated with advance disease and high GI burden [9].

We report a rare case of GI-KS presenting as upper GI bleeding in Sjogren's syndrome individual. Prompt diagnosis and early initiation of therapy are the cornerstones for management of this potentially severe disease.

\section{Case Presentation}

A 70-year-old male had presented with progressively weakness, dizziness, and melena for 1 week. He had past medical history of type 2 diabetes, Sjogren's syndrome and cutaneous Kaposi sarcoma diagnosed by skin biopsy 1 year previously. Physical examinations revealed pallor conjunctiva and erythematous plaques lesions on his lower extremities (Figure 1). Laboratory data showed hemoglobin level of $7.3 \mathrm{~g} / \mathrm{dL}$ and positive result of the fecal occult blood test. The esophagogastroduodenoscopy disclosed numerous 1 to $2 \mathrm{~cm}$, polypoid reddish lesions with ulceration in the stomach (Figure 2 and 3). Biopsy specimens were obtained from the nodular lesions. The pathologic result of biopsy showed moderate chronic inflammation and proliferation of spindle cells with vascular slits and hemorrhage, consistent with Kaposi's sarcoma. Immunohistochemistry stains for human herpes virus- 8 and CD34 were positive, supporting the above diagnosis (Figure 4 and 5). The patient received blood transfusion and proton-pump inhibitor administration. Systemic treatment with doxorubicin was recommended but the patient hesitated the side effects of chemotherapy. His melena and symptoms related to anemia temporarily improved after conservative medical treatment.

\section{Discussion}

In total, 4 forms of KS have been described. As HHV-8 has been detected in all 4 forms of Kaposi sarcoma. Classic KS typically occurs in older men of Eastern European and Mediterranean countries. This form is not associated with HIV, but it coincides with an altered immune system and malignant diseases. Endemic or African KS is prevalent in sub-Saharan Africa and is not correlation with immune deficiency. This form is very aggressive and is often found in South Africa in young Bantu children with local or generalized lymphadenopathy. Organ transplant associated KS occurs after solid organ transplantation. This form develops between several months to several years after organ transplantation with immunosuppressive 


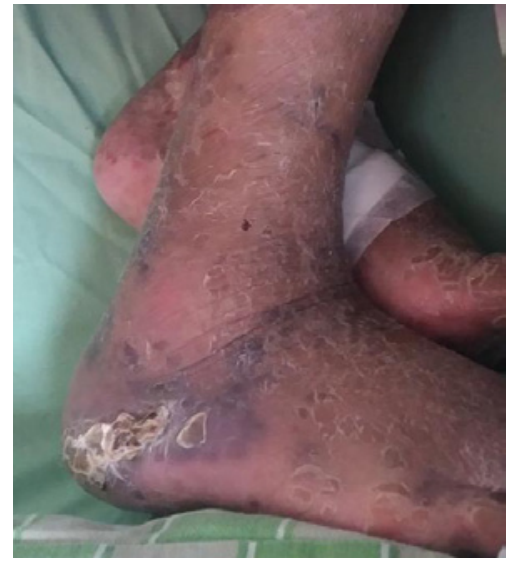

Figure 1: Purple and brown lesions on his lower extremities, performed biopsy 1 year previously.

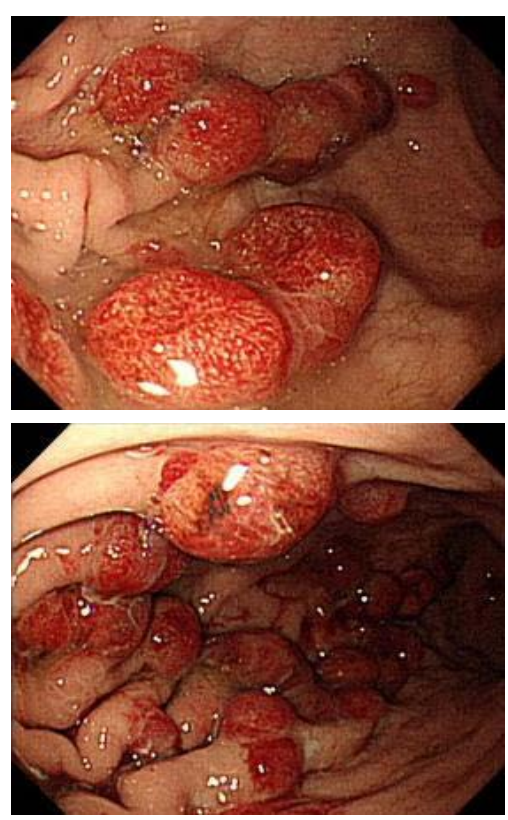

Figure 2 and 3: Dark-reddish polypoid nodule lesions in the stomach.

therapy. Lesions develop on the skin, but in approximately half of the cases, they are also found in internal organs and lymph nodes. AIDS-related KS preferentially affects homosexual or bisexual men compared with women, drug users, or transfusion recipients. Although the rate of AIDS related KS has decreased dramatically since the introduction of highly active antiretroviral therapy (HAART), KS remains the most common malignancy among patients with AIDS [10-12].

KS has long been suspected of having an infectious etiology on the basis of its unusual epidemiology, histopathology, and natural history. Additionally, the virus also modulates innate immune pathways to allow for prolonged survival of the infected cell following primary infection, and during viral latency. The pathogenesis of KS has been linked to human herpesvirus 8 infections. Kaposi's sarcomaassociated herpesvirus (KSHV) pathogenesis also depends on cell extrinsic events such as co-infection with human immunodeficiency

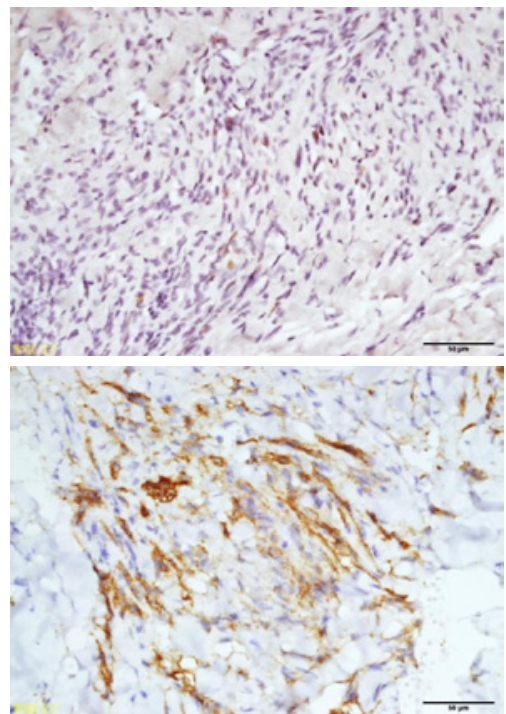

Figure 4 and 5: Immunohistochemical staining revealing expression of human herpes virus-8 (HHV-8) and CD-34.

virus (HIV), degree and status of the systemic host immune response, and other host factors, which modulate the cellular environment [13].

KSHV binding to the endothelial cell surface heparan sulfate is followed by sequential interactions with $\alpha \mathrm{V} \beta 3, \alpha \mathrm{V} \beta 5$, and a3 $\beta 1$ integrins and Ephrin A2 receptor tyrosine kinase (EphA2R). In recent research, it demonstrated that the endosomal Sorting Complexes Required (ESCRT-I) complex Tsg101 protein also involves in the macropinocytosis of KSHV and plays an important role in KSHV trafficking. The Endosomal Sorting Complexes Required for Transport (ESCRT) proteins are recruited to assist in viral entry and trafficking. In addition, KSHV interacting with the cell surface receptors also induces the host transcription factors NF- $\mathrm{kB}$, $\mathrm{Nrf2}$, and ERK1/2 during infection to initiate and modulate viral and host gene expression [14-15].

KS of the gastrointestinal tract is not an uncommon disease in acquired immunodeficiency syndrome and can also occur in immunosuppressed patients $[16,17]$. As reported in the literature, $40 \%$ of patients with confirmed Kaposi's sarcoma will develop GI lesions as well. In the GI tract, the small intestine is affected most frequently, followed by the stomach, esophagus, and colon. Most gastrointestinal lesions might be asymptomatic, but abdominal pain, weight loss, nausea, vomiting, gastrointestinal bleeding, obstruction, malabsorption, or diarrhea are all possible manifestations . Previous reports showed gastrointestinal bleeding occurred in renal transplant recipients with KS of gastrointestinal tract. Acute GI bleeding due to GI-KS in Sjogren's syndrome individual has been infrequently reported in the literature [18-20].

On endoscopic findings of gastrointestinal KS, it is characterized by features such as deeply-red flat, maculopapular, or polypoid lesion. Volcano-like mass with a central umbilication or ulceration may present in some cases $[5,20]$. Diagnostic histopathology demonstrates spindle shaped cells with positive nuclear staining for human herpes virus-8. Immunohistochemistry shows expression of CD34, CD31 and D2-40 [21]. 
Overall, the visceral involvement of the KS is usually associated with poor prognosis. Treatment is usually palliative and aimed primarily at improving symptoms and preventing progression. Options may include antiretroviral medications, radiation therapy, chemotherapy, or combination therapy. Treatment with HAART is recommended in AIDS-related KS. Antiretrovirals may help decrease the proportion of new lesions, promote regression of existing lesions, and improve survival with or without chemotherapy. Among patients with localized cutaneous KS, HAART with either topical gel therapy or local radiation may suffice. However, in patients with extensive mucocutaneous disease or visceral organ involvement, combined HAART and systemic chemotherapy is indicated. Pegylated Liposomal doxorubicin and paclitaxel are the chemotherapeutic agents of choice [22,23].

Other drugs have been studied including antiangiogenic agents (e.g. thalidomide) and immunomodulators (e.g. retinoid compounds). All have shown suboptimal response rates. Antiviral agent cidofovir, had also been investigated in small trials suggesting it could be as a possible alternative therapy. While targeting mTOR and VEGF are promising therapeutic strategies, the inhibition of other up regulated pathways in KS have been the focus of recently completed clinical trials. Finally, another promising therapeutic strategy for KS is the inhibition of matrix metalloproteinases (MMPs). MMPs are endopeptidases that assist in angiogenesis via degradation of extracellular matrix [23]. The ability to molecularly target intricate pathways in cancer has been transformed from a hopeful idea to a definitive reality.

\section{Conclusion}

KS of the GI tract is far more common than originally thought, but the majority of patients are asymptomatic and remain undiagnosed. KS with gastrointestinal involvement should be considered in the differential diagnosis of gastrointestinal bleeding. Therefore, clinicians always maintain a high index of suspicion, especially in patients with AIDS and some immunosuppressive patients.

\section{References}

1. Dittmer DP, Damania B. Kaposi sarcoma associated herpesvirus pathogenesis (KSHV)--an update. Curr Opin Virol. 2013; 3: 238-244.

2. Sullivan RJ, Pantanowitz L, Casper C, Stebbing J, Dezube BJ. Epidemiology, pathophysiology and treatment of Kaposi sarcoma-associated herpesvirus disease: Kaposi sarcoma, primary effusion lymphoma, and multicentric Castleman disease. Clin Infect Dis. 2008; 47: 1209-1215.

3. Beral V, Peterman TA, Berkelman RL, Jaffe HW. Kaposi's sarcoma among persons with AIDS: a sexually transmitted infection? Lancet. 1990; 335: 123128.

4. Buchacz K, Baker RK, Palella FJ, Chmiel JS, Lichtenstein KA, Novak RM, et al. AIDS-defining opportunistic illnesses in US patients, 1994-2007: A cohort study. AIDS. 2010; 24: 1549-1559.

5. Nagata N, Shimbo T, Yazaki H, Asayama N, Akiyama J, Teruya K, et al Predict clinical factors in the Diagnosis of Gastrointestinal Kaposi's Sarcoma and Its Endoscopic Severity. PLoS One. 2012; 7: e46967.
6. Hancock S, Pfau P. Kaposi sarcoma involving the gastrointestinal tract: review. Gastroenterol Hepatol. 2010; 6: 463-464.

7. Ioachim HL, Adsay V, Giancotti FR, Dorsett B, Melamed J. Kaposi's sarcoma of internal organs. A multiparameter study of 86 cases. Cancer. 1995; 75: 1376-1385.

8. Querido S, Sousa HS, Pereira TA, Birne R, Matias P, Jorge C, et al. Gastrointestinal Bleeding and Diffuse Skin Thickening as Kaposi Sarcoma Clinical Presentation. Case Rep Transplant. 2015; 2015: 424508.

9. Rodriguez S, Zapatier J, Allende D, Schneider A. Kaposi's Sarcoma: An Unusual Cause of Gastric Outlet Obstruction. ACG Case Rep J. 2013; 1: 19-21.

10. Mocroft A, Kirk O, Clumeck N, Gargalianos-Kakolyris P, Trocha H, Chentsova $\mathrm{N}$, et al. The changing pattern of Kaposi sarcoma in patients with HIV, $1994-$ 2003: the Euro SIDA Study. Cancer. 2004; 100: 2644-2654.

11. Levine AM, Tulpule A. Clinical aspects and management of AIDS-related Kaposi's sarcoma. Eur J Cancer. 2001; 37: 1288-1295.

12. Stebbing J, Sanitt A, Nelson M, Powles T, Gazzard B, Bower M. A prognostic index for AIDS-associated Kaposi's sarcoma in the era of highly active antiretroviral therapy. Lancet 2006; 367: 1495-1502.

13. Ganem D. KSHV infection and the pathogenesis of Kaposi's sarcoma. Annu Rev Pathol. 2006; 1: 273-296.

14. Kumar B, Dutta D, Iqbal J, Ansari MA, Roy A, Chikoti L, et al. ESCRT-I Protein Tsg101 Plays a Role in the Post-macropinocytic Trafficking and Infection of Endothelial Cells by Kaposi's Sarcoma-Associated Herpesvirus. PLoS pathog. 2016 ; 12: e1005960.

15. Kumar B, Chandran B. KSHV Entry and Trafficking in Target Cells-Hijacking of Cell Signal Pathways, Actin and Membrane Dynamics. Viruses. 2016; 8.

16. Friedman SL, Wright TL, Altman DF. Gastrointestinal Kaposi's sarcoma in patients with acquired immune deficiency syndrome: Endoscopic and autopsy findings. Gastroenterology. 1985; 89: 102-108.

17. Balachandra B, Tunitsky E, Dawood S, Hings I, Marcus VA. Classic Kaposi's sarcoma presenting first with gastrointestinal tract involvement in a HIVnegative Inuit male--a case report and review of the literature. Pathol Res Pract. 2006; 202: 623-626.

18. Mansfield SA, Stawicki SP, Forbes RC, Paradimos TJ, Lindsey DE. Acute upper gastrointestinal bleeding secondary to Kaposi sarcoma as initial presentation of HIV infection. J Gastrointestin Liver Dis. 2013; 22: 441-445.

19. Attia S, Dezube BJ, Torrealba J, Sosman JM, McHaffie DR, Pfau PR, et al AIDS-related Kaposi's sarcoma of the gastrointestinal tract. J Clin Oncol. 2010; 28: e250-e251.

20. Lee AJ, Brenner L, Mourad B, Monteiro C, Vega KJ, Munoz JC. Gastrointestinal Kaposi's sarcoma: Case report and review of the literature. World J Gastrointest Pharmacol Ther. 2015; 6: 89-95.

21. Kaiserling E. Immunohistochemical identification of lymph vessels with D2-40 in diagnostic pathology. Pathologe. 2004; 25: 362-374.

22. Di Lorenzo G, Konstantinopoulos PA, Pantanowitz L, Di Trolio R, De Placido S, Dezube BJ. Management of AIDS-related Kaposi's sarcoma. Lancet Oncol. 2007; 8: 167-176.

23. Sullivan RJ, Pantanowitz L, Dezube BJ. Targeted therapy for Kaposi sarcoma. BioDrugs. 2009; 23: 69-75.
Austin J Gastroenterol - Volume 4 Issue 1 - 2017 ISSN : 2381-9219 | www.austinpublishing group.com Tien-Yu Huang et al. (C) All rights are reserved
Citation: Huang M-Y, Liu F-C, Gao H-W and Huang T-Y. Metastatic Kaposi's Sarcoma Causing Gastrointestinal Bleeding: Report of an Unusual Case. Austin J Gastroenterol. 2017; 4(1): 1074. 